Géocarrefour, $\mathrm{n}^{\circ}$ 84/4, 2009, pp. 229-239

Géohistoire/géo-histoire : quelles méthodes pour quel récit?

\title{
Sources historiques et problématiques de recherche en géographie des risques naturels
}

\author{
Jean-Marc Antoine*, Bertrand Desailly*, Anne Peltier*
}

\section{Introduction}

Les sources historiques, du moins dans les pays et régions où leur production a été abondante sur des durées assez longues, sont une contribution majeure à une bonne appréciation des situations de risque naturel. L'aléa de très forte intensité est souvent éloigné dans le temps de plusieurs décennies, voire de plusieurs siècles dans le cas de séismes, et le rappel ou la révélation de son existence, associés à une reconstitution aussi précise que possible de son déroulement et de son emprise spatiale, sont susceptibles d'orienter judicieusement les choix d'aménagement contemporains. Ainsi, dans le cas des Plans de Prévention des Risques d'Inondation (PPRI), la cartographie des zones inondables prend normalement en compte les plus hautes eaux connues (PHEC). Face à une opinion publique oublieuse et fréquemment sceptique, le document ancien est à même de fournir la preuve de la réalité d'une menace et de faire accepter des mesures parfois contraignantes. La valeur pédagogique d'une photographie, prise il y a un siècle, d'un village de montagne traversé par une crue torrentielle, n'est plus à démontrer. Elle rejoint celle des repères de crues sur les façades des bâtiments ou des monuments commémoratifs de grandes catastrophes.

Encore faut-il savoir chercher, interpréter, exploiter ces documents anciens, ce qui ne s'improvise pas. Parce qu'ils disposaient de ce savoir-faire, universitaires et chercheurs, notamment des géographes, ont été fréquemment sollicités par les organismes publics en charge de la gestion du risque. Avec pour corolaire une importante production, en particulier sous forme cartographique, relevant de la recherche appliquée. Au-delà de cet aspect, on peut s'interroger sur la contribution des archives, au cours des années 1980 et 1990, à l'émergence de nouvelles problématiques et à la structuration thématique de la recherche en géographie des risques naturels. La démarche est aujourd'hui, en quelque sorte, inversée. Une plus grande familiarité avec les différents types de sources permet d'en mesurer rapidement l'intérêt, mais aussi les limites, dans le cadre de réflexions portant notamment sur les relations entre histoire des aléas et histoire de l'environnement, sur l'évolution des représentations du risque, sur l'analyse des politiques de prévention et de protection ou encore sur la construction de la vulnérabilité.

\section{La redécouverte des sources historiques}

\subsection{Un contexte propice}

La thématique des risques naturels mobilise, dans les années 1980 et au début des années 1990, un nombre croissant de chercheurs en sciences de la Terre et en sciences humaines, dans un climat politique et social de forte demande de connaissance sur le sujet. Une activité législative soutenue, entretenue par la répétition de plusieurs événements à caractère catastrophique, se traduit notamment par l'instauration, en 1984, des Plans d'Exposition aux Risques (PER), puis en 1995, des Plans de Prévention des Risques (PPR). Les deux procédures incluent la réalisation de cartes des événements historiques survenus dans les espaces concernés (Garry, 1996). L'intérêt de l'information historique pour l'estimation des situations actuelles de risque est donc clairement souligné par les pouvoirs publics, alors même que les services de l'Etat chargés de mettre en œuvre les nouvelles politiques de prévention ne sont pas nécessairement les mieux armés pour accéder à cette information. 
Le contexte semble donc favorable au développement de relations avec ceux qui, au sein du monde universitaire, s'avèrent familiers des documents anciens. Pourtant, peu nombreux sont les historiens à s'engager franchement dans cette voie. La fructueuse collaboration de J. Vogt avec le BRGM, sur le thème de la sismicité historique de la France, fait un peu figure d'exception (Vogt, 1979). Le positionnement des géographes, moins à l'aise sans doute que leurs collègues historiens face aux sources écrites ou iconographiques, particulièrement les plus anciennes, mais possédant une bonne connaissance des processus physiques, aptes par là-même au dialogue avec les ingénieurs forestiers, les hydrauliciens, les géologues, etc., attentifs enfin à une spatialisation des phénomènes recherchée par les services de l'Etat, leur permet de profiter davantage de cette opportunité.

Sur le plan de la recherche scientifique, la période est par ailleurs marquée par un fort développement des pratiques interdisciplinaires autour des « questions vives » relevant du champ en plein essor de l'environnement. Programmes interdisciplinaires de recherches du CNRS à partir de 1979 (PIREN Histoire de l'environnement), lancement de revues (Nature, Sciences, Sociétés), constitution de groupes de chercheurs (Groupe d'histoire des forêts françaises) accompagnent le mouvement. Parmi les sujets de recherche, risques naturels et catastrophes occupent une place privilégiée, constituant le thème central de plusieurs colloques (L'Aiguat del 40, Vernet-les-Bains, 1990 ; Les catastrophes naturelles dans l'Europe médiévale et moderne, Flaran, 1993). Mais le sujet est aussi fortement présent dans nombre de manifestations scientifiques, à travers par exemple l'étude du rôle des ingénieurs dans l'aménagement des cours d'eau (Le fleuve et ses métamorphoses, Lyon, 1992). Il faudrait encore ajouter à cette liste la parution de numéros spéciaux de revues (Sources - Travaux historiques, Histoire des catastrophes naturelles, 1993). En l'espace de quelques années, les occasions se multiplient de rencontres entre géographes et historiens, débouchant sur des emprunts méthodologiques et thématiques réciproques.

Par le biais de l'environnement, la géographie renoue avec un matériau - les archives - et une échelle temporelle - l'échelle pluriséculaire - quelque temps un peu délaissés, en même temps que se distendait la relation privilégiée entre géographie et histoire au profit d'autres rapprochements disciplinaires. Car il s'agit bien de la redécouverte, avec de nouvelles problématiques et de nouvelles méthodes, de sources souvent largement exploitées par quelques figures de la géographie classique. Dans sa thèse sur le Val de Loire publiée en 1933, R. Dion explore longuement l'évolution des relations entre occupation du sol, risque d'inondation et dispositifs de défense contre les eaux. Il en tirera une Histoire des levées de la Loire, parue en 1961. Dans la longue synthèse que M. Pardé consacre, au milieu des années 1950, aux grandes crues dans le bassin de la Garonne, les références historiques sont également nombreuses (Pardé, 1953). L'imposante documentation réunie au cours de sa carrière par le père de la potamologie constitue du reste aujourd'hui un fonds d'un grand intérêt, inventorié et classé en 1992 et 1993. M. Pardé avait par exemple recueilli, à la suite de la crue d'octobre 1940 dans les Pyrénées-Orientales, les témoignages des instituteurs en poste dans le département. G. Soutadé a montré le parti que l'on pouvait tirer d'un tel matériau (1993).

Il reste que, pour les chercheurs géographes dont la formation s'est déroulée au cours des années 1970 à 1980, la fréquentation des dépôts d'archives ne va pas forcément de soi. Nouveaux lieux de recherche, nouvelles pratiques aussi, nécessitant de se familiariser avec un mode de classement spécifique, avec des outils, inventaires ou répertoires, alors non encore informatisés. Les enseignements de second et de troisième cycle en géographie n'abordent guère ce domaine et, si la démarche intellectuelle de la critique des sources est à peu près intégrée, l'essentiel de l'apprentissage s'effectue sur le tas, avec ses inévitables pertes de temps et possibles erreurs d'interprétation des documents, faute d'une parfaite connaissance du contexte de leur production.

Rapidement cependant, quelques grandes familles de sources exploitables sont identifiées. Nombre d'entre elles sont susceptibles d'alimenter une réflexion dans plusieurs directions: histoire des aléas, relations entre risque et occupation de l'espace, évolution des politiques de gestion du risque, etc. Les photographies prises à la fin du XIX ${ }^{\mathrm{e}}$ siècle par les forestiers du service 
de Restauration des Terrains en Montagne (RTM), dans les Alpes, les Pyrénées ou le sud du Massif Central, témoignent d'une activité érosive et torrentielle visiblement plus marquée qu'aujourd'hui, et se prêtent bien à l'exercice désormais classique du couple de clichés pris à un siècle d'intervalle. Elles sont aussi, à travers le choix des cadrages, des lieux de prise de vue, à la fois légitimation d'une action rencontrant localement de fortes oppositions et partie prenante d'un discours de crise - la dégradation des montagnes - qui traverse l'ensemble du XIX ${ }^{\mathrm{e}}$ siècle, et même au-delà.

En raison de leur richesse et de leur facilité relative d'accès, les archives de l'administration des Ponts et Chaussées, rassemblées pour l'essentiel dans la sous-série $\mathrm{F}^{14}$ des Archives Nationales et la série $S$ des Archives Départementales, sont parmi les premières visitées. Rapports et mémoires rédigés par des générations d'ingénieurs en constituent la plus grande part, presque systématiquement accompagnés de cartes, profils, plans, décrivant notamment des travaux de redressement et d'endiguement des cours d'eau. Il y avait là matière à réflexion sur l'enchaînement des dynamiques fluviales et sur le rôle joué par les grandes crues, à travers leur effet érosif ou les travaux qu'elles ont suscités a posteriori.

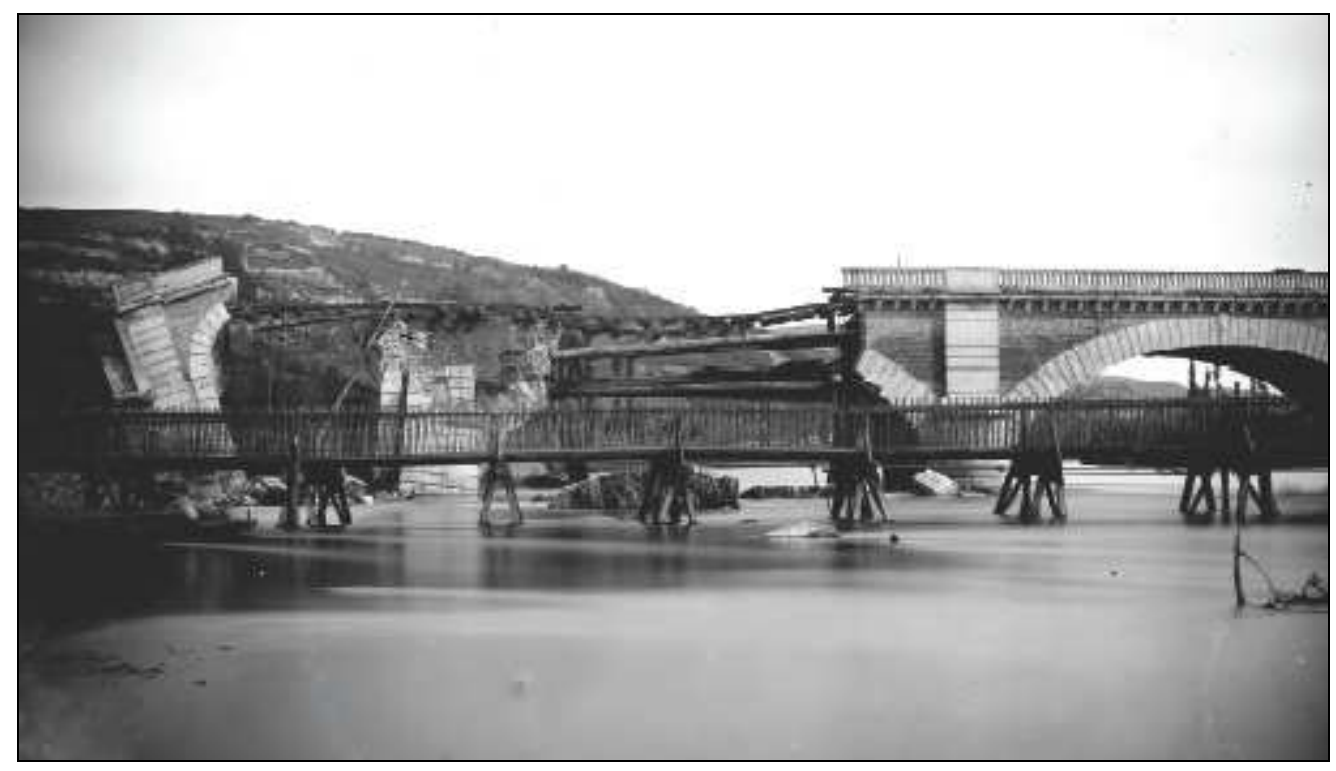

Figure 1. Les dégâts de la crue du 23 juin 1875 à Toulouse. Fonds Eugène Trutat, Muséum d'Histoire Naturelle de Toulouse, Banque d'images des patrimoines et territoires

\subsection{Un usage désormais largement banalisé}

Thèses de doctorat et publications variées ont clairement montré, au milieu des années 1990, le parti que l'on peut tirer de l'information historique dans la connaissance des situations de risque naturel. La recherche appliquée est stimulée par une demande toujours très forte émanant des pouvoirs publics: constitution de bases de données événementielles dans un cadre départemental ou régional, réalisation de cartes d'historicité des phénomènes à petite et moyenne échelle, mise en œuvre progressive des PPR. La recherche fondamentale quant à elle voit émerger de nouvelles problématiques, autour par exemple de la question de la vulnérabilité ou de l'inscription du risque dans les paysages.

Les chercheurs bénéficient d'un contexte plus favorable que quelques années auparavant. L'accès aux sources est facilité par l'informatisation progressive des répertoires et inventaires, ainsi que par la publication de quelques guides (Corvol, 1999 et 2003). De nombreux documents encombrants ou fragiles sont numérisés : cadastres napoléoniens, collections de photographies anciennes... Par exemple, la collection Eugène Trutat, constituée de photographies sur plaques 
de verre réalisées dans le dernier quart du XIX ${ }^{\mathrm{e}}$ siècle, qui renferme de précieux clichés pris après l'inondation catastrophique du 23 juin 1875 à Toulouse ou du 3 juillet 1897 dans les Pyrénées centrales (fig. 1). Fait significatif: des «classiques » parmi les sources bibliographiques anciennes font l'objet de rééditions, au premier chef le travail monumental - et cependant lacunaire dans le recensement des événements - de M. Champion, Les inondations en France depuis le Vle siècle (18621868, réédition 2000), ou encore l'Etude sur les torrents des Hautes-Alpes d'A. Surrel (1841, $2^{\mathrm{e}}$ édition 1870, réédition 2002). Face à des documents globalement plus accessibles, les chercheurs sont enfin mieux armés, l'exploitation des archives faisant désormais couramment partie de l'arsenal méthodologique présenté aux étudiants dans les formations de niveau master en géographie de l'environnement.

Les investigations portent désormais sur un éventail plus large de sources archivistiques, faisant progresser les connaissances notamment dans la spatialisation des phénomènes. La confrontation des dossiers d'indemnisation de sinistrés et des plans cadastraux autorise des cartographies à des échelles très fines, tant des dommages aux biens que des effets géomorphologiques des crues ou de la direction des écoulements (Jacob, 1997; Meschinet de Richemond, 1997). Le dépouillement de la presse régionale et locale est aussi susceptible de fournir des informations précises - film des événements heure par heure, rues et places inondées, victimes et circonstances de leur décès, etc. -, moyennant quelques précautions d'interprétation. Ce type de documents constitue par ailleurs un support privilégié pour l'étude des représentations $\mathrm{du}$ risque, domaine propre à susciter rapprochements et confrontations entre approches historique, sociologique et géographique du risque (Revue de Géographie Alpine, Représentation des risques naturels en montagne, 1998). Les limites à cet élargissement de la base de collecte sont à rechercher du coté des documents les plus anciens, antérieurs au XVII ${ }^{\mathrm{e}}$ siècle, rarement exploités directement par les géographes. Plusieurs raisons peuvent être invoquées : difficulté de lecture des textes médiévaux en latin, défaut de formation en paléographie, « rendement » incertain des sources... Des collaborations avec les historiens s'avèrent encore ici indispensables.

En raison de l'importance des enjeux d'aménagement contemporains et de la grande richesse de la documentation historique, les villes et leur environnement proche demeurent un terrain d'étude privilégié (Cœur, 2003), le recours aux SIG renouvelant le traitement de l'information (Combe, 2008). Des approches plus classiques sont mises en œuvre dans des synthèses à l'échelle régionale, par exemple pour le Languedoc et le Roussillon (Antoine et al., 2001a). Sur le plan thématique, l'évolution des politiques de gestion du risque continue d'occuper une place importante, qu'elle soit abordée dans le cadre de monographies ou bien d'études comparatives entre plusieurs pays d'Europe (Peltier, 2005). Les travaux géohistoriques sur les risques et catastrophes naturelles trouvent par ailleurs une nouvelle résonnance avec le développement important des recherches sur l'histoire du climat (Antoine, 2009).

\section{Données anciennes et histoire des aléas}

\section{Un symptôme de dynamiques environnementales}

L'approche géohistorique des catastrophes et des risques naturels s'est développée au cours des années 1980 en parallèle d'une histoire plus globale de l'environnement biophysique, laquelle est notamment sous-tendue par le paradigme d'une co-construction des environnements actuels par l'homme et la nature. Par conséquent, c'est tout naturellement que, pour mieux comprendre les dynamiques contemporaines de l'environnement comme celles des risques naturels, s'est imposée une approche historique. Par ailleurs, un des principaux intérêts de la mise en perspective de la dynamique des catastrophes naturelles avec les dynamiques environnementales réside dans le différentiel temporel qui les sépare mais aussi les relie étroitement : les premières ne 
sont en fait qu'un temps fort des secondes, une sorte de signal, de marqueur ponctuel d'autres dynamiques aux temporalités beaucoup plus longues, qu'il s'agisse de dynamiques environnementales s.s. (dynamiques végétales, climatiques et hydroclimatiques...) ou de dynamiques sociales (occupation et usage des sols, politiques de protection, perception sociale de l'environnement...).

Parmi les premières recherches sur l'histoire des aléas, celles menées au sein du laboratoire GEODE UMR 5602-CNRS (ex-CIMA) ont porté sur la reconstitution de chronologies événementielles centrées sur le massif pyrénéen et son piémont (Desailly, 1990 ; Métailié, 1991 ; Antoine, 1992). A peu près au même moment, les Alpes ont fait l'objet de travaux similaires de la part de géographes (Miramont et al., 1997 ; Baraillé, 2001), mais aussi de l'historien G. Pichard (1995). On dispose avec ces chronologies d'une bonne perspective historique, parfois depuis le $\mathrm{XIV}^{\mathrm{e}}$ siècle, de la marche des phénomènes catastrophiques, inondations et crues torrentielles principalement. Elles ont mis en lumière une évolution rythmée des phénomènes, alternant temps de crises et temps de répits, certains étant déjà connus à l'image de la crise torrentielle de la seconde moitié du XIX ${ }^{\mathrm{e}}$ siècle (Métailié, 1987), d'autres étant découverts à cette occasion (fin du $\mathrm{XVIII}^{\mathrm{e}}$ siècle) (fig. 2). Au-delà des chronologies, dont l'un des intérêts majeurs résidait dans leur mise en parallèle avec les «attitudes» sociales face aux risques, c'est tout un travail de recensement et de caractérisation qualitative et semi-quantitative ou quantitative des aléas qui a été mené, permettant de reconstituer la dynamique précise des inondations et crues torrentielles, mais aussi des mouvements de terrain (Fanthou \& Kaiser, 1990) ou encore de la torrentialité sur les versants (Lahousse, 1997), replacés en outre dans leurs contextes socio-écologiques historiques.

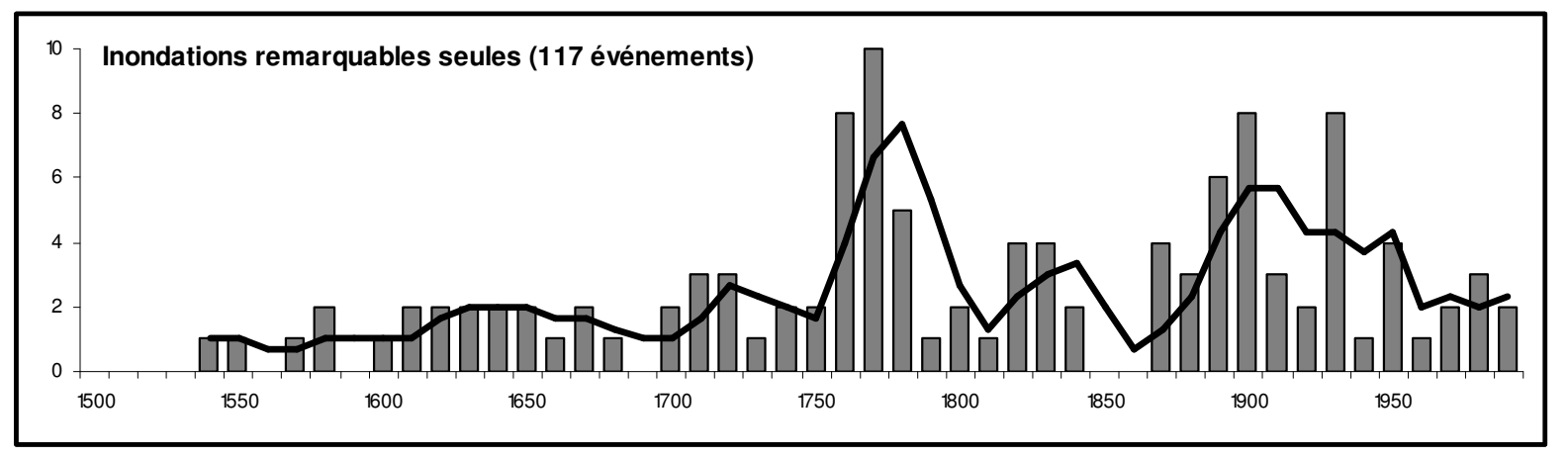

Figure 2. Les inondations remarquables dans les Pyrénées (1500-2000) (Antoine, 2009)

A peu près au même moment, d'autres chercheurs ont mobilisé les inondations et les crues torrentielles en tant qu'indicateurs d'autres dynamiques environnementales. Ainsi de J.-P. Bravard et de ses élèves qui, en démontrant et analysant les ressorts de la métamorphose fluviale affectant les cours d'eau alpins depuis le XVII ${ }^{\mathrm{e}}$ siècle voire même avant, interprètent les grandes crues ou les périodes de grandes crues comme des moments-clés de cette métamorphose (Peiry, 1988 ; Salvador, 1991 ; Gautier, 1992 ; Cubizole, 1994). Ce type de recherche a embrassé par la suite des temps plus longs, s'appuyant alors sur des sources et des méthodes géoarchéologiques (ArnaudFassetta, 1998 ; Miramont, 1998).

En mettant en avant une évolution des phénomènes scandée par des rythmes d'amplitude multidécennale à séculaire, l'histoire des aléas a enfin alimenté une problématique environnementale promise à un bel avenir, l'histoire du climat. Sur cette question, les historiens ont été précurseurs, avec E. Le Roy Ladurie (1967) mais surtout Ch. Pfister (1988) dont les travaux depuis plus de vingt ans lient directement histoire des aléas hydrométéorologiques et histoire du climat. Cette problématique est l'objet aujourd'hui d'un intérêt renouvelé dans le 
contexte du changement climatique et de ses effets supposés sur les aléas atmosphériques, hydrologiques et glaciaires. Les recherches s'attachent notamment à mieux comprendre de ce point de vue la période du Petit Age Glaciaire ${ }^{1}$.

Dans ce domaine de l'histoire des aléas, envisagée pour elle-même ou inféodée à d'autres problématiques environnementales, les investigations historiques se sont appuyées sur des séries d'archives particulièrement productives au sein des Archives Départementales : rapports circonstanciés d'ingénieurs du Roi puis des Ponts \& Chaussées ou des Eaux \& Forêts, avec cotes de crues, plans et photographies (séries $\mathrm{C}, \mathrm{S}$ et $\mathrm{P}$ ) ; rapports de gendarmerie, demandes et états de vérification de pertes, de dégrèvements et de remise d'impositions (séries $\mathrm{C}$ et $\mathrm{M}$ ). Selon les problématiques, d'autres sources peuvent néanmoins être utiles : ainsi des cartes anciennes et des cadastres conservés dans les séries $\mathrm{Fi}$ et $\mathrm{P}$ pour les travaux sur l'évolution de la morphodynamique fluviale (Peiry, 1989), ou encore des chroniques, des livres de raison, des observations météorologiques pour les relations entre histoire des catastrophes et histoire du climat (séries E et J). Notons également qu'à partir du XIX ${ }^{\mathrm{e}}$ siècle apparaissent deux autres types de sources qui, malgré la nécessité d'un examen critique plus approfondi que pour les documents d'archives publiques, sont susceptibles de fournir des informations. Il s'agit, d'une part, des nombreux ouvrages, opuscules ou articles d'érudits locaux s'attachant à reconstituer l'histoire des inondations, voire des catastrophes naturelles, sur le modèle des six tomes de M. Champion consacrés aux Inondations en France depuis le VIe siècle (1862-1868)2. D'autre part, la première moitié du $\mathrm{XIX}^{\mathrm{e}}$ siècle voit le développement de la presse locale, à parution quotidienne, hebdomadaire ou mensuelle, source prolifique d'informations sur les événements venant de se produire : 350 titres ont ainsi été recensés aux Archives Départementales du Gers, couvrant ensemble la période allant de 1814 à aujourd'hui (Antoine, Desailly, 2000) (fig. 3). La seconde moitié du XIX siècle voit également émerger un troisième type de source, a priori plus objectif que les précédents : les photographies, qu'elles émanent d'amateurs éclairés, d'administrations tel le service RTM, ou encore des premiers éditeurs de cartes postales (série Fi).

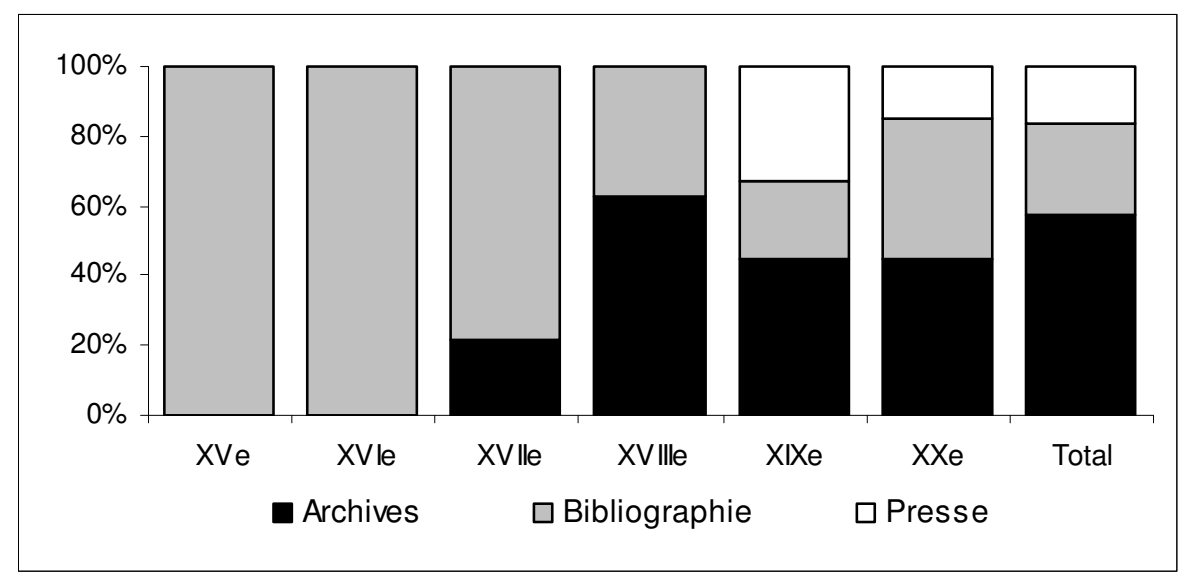

Figure 3. Les sources historiques relatives aux inondations dans le département du Gers. (Antoine, Desailly, 2000)

\footnotetext{
1 - Cf. la table-ronde «Le Petit Age Glaciaire dans le sud de la France », organisée à Lattes en 2007 et dont les actes sont sous presse dans la revue Archéologie du Midi Médiéval.

2 - Parmi de nombreuses références, on peut citer R. Guiraud de Saint-Marsal, 1856, Mémoire sur les inondations occasionnées par les crues de la Têt et de la Basse aux environs de Perpignan ", Bulletin de la Société Agricole, Scientifique et Littéraire des Pyrénées-Orientales, tome X, ou encore L. Gaussen, 1937, Le Vidourle et ses vidourlades, Nîmes, Editions Méridionales, 200 p.
} 


\subsection{Des applications opérationnelles}

Parmi les ressorts du développement d'une approche géohistorique des risques naturels se place aussi la demande sociale et institutionnelle, émanant notamment des gestionnaires territoriaux du risque (ministères, services déconcentrés de l'Etat). Leur intérêt pour l'information historique n'est pas récent. Depuis l'Ancien Régime, l'évocation des catastrophes passées est une constante dans l'argumentation des ingénieurs, des administrateurs territoriaux, des édiles locaux, voire des particuliers. Elle pouvait avoir plusieurs objectifs : étalonner un événement venant de se produire, le faire accepter plus facilement par les sinistrés, justifier des mesures de protection/correction, motiver une demande de non-imposition face à la récurrence des phénomènes... Au XIX ${ }^{\mathrm{e}}$ siècle, tout bon rapport d'ingénieur des Ponts \& Chaussées ou des Eaux \& Forêts à propos d'aménagements à réaliser (périmètres de reboisement, endiguement...), de réglementation de l'usage des sols (agropastoralisme, usage des eaux et des bois, aménagement des berges...) ou encore d'un événement venant de se produire, ne manque pas de faire référence à des antécédents historiques du même ordre. C'est ainsi à une demande du maire d'Agen projetant d'aménager les bords de Garonne que l'on doit l'inventaire le plus complet et le plus critique des crues de la Garonne à Toulouse et Agen, rédigé en 1918 par F. Galabert, conservateur des Archives Municipales de Toulouse. Au XX ${ }^{\mathrm{e}}$ siècle, ce qui n'était souvent qu'une figure de style s'est peu à peu institutionnalisé, pour être aujourd'hui inscrit sur les tables de la loi.

En 1935, le Plan des Surfaces Submersibles (PSS) est le premier document règlementaire de zonage des risques à tenir compte de l'information historique (Garry, 1994), en délimitant sur des plans au 1/25 000 les PHEC, ou Plus Hautes Eaux Connues. De la même manière, les Cartes de Localisation Probable des Avalanches (CLPA), réalisées à partir des années 1970 par le CEMAGREF, sont partiellement conçues à partir des événements historiques observés. En 1984, c'est au tour du Plan d'Exposition aux Risques Naturels Prévisibles (PER) de prévoir dans le rapport final une Carte Informative des Crues Historiques (CICH) montrant l'extension et les dommages des plus grandes crues connues, procédure maintenue lors de la transformation des PER en PPR par la loi Barnier de 1995. Parallèlement et moins formellement, les documents internes et les études des services gestionnaires (Ministères, DDE, RTM, DDAF, DIREN...) intègrent aussi l'information historique dans leurs analyses ${ }^{3}$. Dernièrement, les divers documents de cartographie informative, tels les Atlas des Zones Inondables, ont également mobilisé les données historiques.

L'intérêt de cette information historique pour l'analyse des aléas par les services gestionnaires, en particulier sous ses formes photographique et cartographique, a été démontré (Lang et al., 1998 ; Cœur et al., 2002). Elle permet notamment de localiser les sites à risque, d'approcher la fréquence et l'intensité des aléa sur ces sites, de valider ou d'ajuster les modèles hydrauliques ou trajectographiques (extension des champs d'inondation, des coulées d'avalanches, des chutes de blocs), de sensibiliser et de convaincre les différents acteurs (élus, particuliers...) de la nécessaire attention devant être portée aux risques naturels.

Les sources historiques les plus intéressantes dans ce contexte sont identiques à celles citées ci-dessus. On précisera cependant que, outre dans les dépôts d'archives publiques, beaucoup de ces sources sont souvent conservées aussi par les services eux-mêmes. Par ailleurs, ceux-ci disposent parfois, et en exclusivité, de séries de données débutant pour les plus anciennes dans les années 1880, et qui peuvent faire l'objet de traitements statistiques: hauteurs d'eau aux stations hydrométriques, mesures météorologiques continues ou relatives à un événement particulier, recensement systématique des avalanches (Enquête Permanente sur les Avalanches de l'ONF/RTM)...

\footnotetext{
3 - C'est le cas par exemple des Etudes sur les crues fluvio-torrentielles et le ruissellement urbain menées en 1993 par la Délégation aux Risques Majeurs du Ministère de l'Environnement sur 24 départements du sud de la France, ou encore des Enquêtes de programmation des actions RTM (Services de Restauration des Terrains en Montagne, ONF/DDAF) lancées dans les années 1980-1990 dans les Alpes et les Pyrénées.
} 
Finalement, au-delà de l'acquisition de données factuelles, quantitatives, et de la constitution de chronologies événementielles utilisables dans d'autres problématiques de recherche, ce genre de collaboration a été aussi l'occasion pour les chercheurs de mener diverses réflexions : sur les modalités d'intégration des données historiques aux données plus récentes validées (Naulet, 2002 ; Payrastre, 2005), sur les représentations cartographiques de la dynamique historique des phénomènes (Lahousse, 1994 ; Antoine et al., 2001b ; Léone \& Vinet, 2008), et enfin sur les bases d'une méthodologie de collecte, d'analyse critique, d'utilisation et de stockage des données (Antoine, Desailly, 2000). De fait aujourd'hui, un grand nombre de données historiques sur les aléas naturels font l'objet de bases de données informatisées telles la BD RTM (aléas montagnards) ou celles du BRGM${ }^{4}$.

\section{Données anciennes et histoire des relations entre risques et sociétés}

\subsection{Les représentations des risques}

La relation entre sociétés et aléas passe par la construction d'un discours explicatif du risque, qui attribue aux phénomènes dommageables des causes qui ont beaucoup varié dans l'histoire (Favier, Granet-Abisset, 2005). Les représentations savantes du risque sont sans doute les plus aisément accessibles puisqu'elles sont issues d'une littérature préscientifique ou technique qui a été conservée dans les archives. Les mémoires savants des XVIIe et XVIIIe siècles sur les causes des catastrophes (Briffaud, 1993), puis, au XIXe siècle, les rapports des ingénieurs des Eaux et Forêts et des Ponts et Chaussées sur les phénomènes observés (Culmann et al., 1869 ; Surrel, 1841), les innombrables discours et opuscules publiés constituent un matériau de premier choix pour comprendre les représentations du risque, lesquelles sont au demeurant désormais bien connues, surtout pour les deux derniers siècles. En particulier, le discours sur le déboisement des montagnes et ses conséquences en matière de torrentialité a été amplement étudié et plusieurs auteurs ont mis en évidence ses non-dits (Corvol, 1987 ; Larrère et al., 1979). Signalons à ce propos tout l'intérêt d'une analyse comparative de la production du discours savant sur les crues au XIXe siècle, analyse qui démontre d'importants phénomènes de capillarité entre pays voisins et la constitution d'un corpus doctrinal commun par-delà les frontières (Suisse, Italie, France, Allemagne, Autriche) et par-delà les massifs (Alpes, Pyrénées) (Chalvet, 2000 ; Peltier, 2005 ; Subotsch, 1999). La difficulté de l'analyse tient alors, outre les questions linguistiques, à ce que l'organisation des archives peut être sensiblement différente d'un pays à l'autre. En ce qui concerne la Suisse et certaines régions d'Italie du Nord, toutefois, l'influence française (plus précisément : napoléonienne) dans l'organisation administrative contribue à faciliter le travail, une bonne partie des données étant fournie par les rapports des ingénieurs des Eaux et Forêts.

Les représentations populaires sont quant à elles plus difficiles à saisir, dans la mesure où les populations, qu'elles soient considérées comme victimes ou comme sources des dommages, ont laissé peu de textes. Elles sont souvent connues indirectement, soit par les traces qui restent aujourd'hui de leur culture, soit par le regard que portaient sur elles les élites de l'époque. Les ethnologues, les historiens, ont ainsi travaillé sur les contes et les légendes qui sont parvenus jusqu'à nous (Reyt, 2000 ; Schüle, 1992 ; Samivel, 1984) ; ils ont également étudié la toponymie, révélatrice d'un certain rapport au monde (Berot, 1998 ; Bessat, Germi, 1993). Dans certains cas toutefois, des données de première main ont pu être analysées et fournissent des informations précieuses sur les relations des populations à leur environnement (Schoeneich, Busset-Henchoz, 1998). Curieusement, et en dépit de l'avancée des connaissances scientifiques, les données anciennes permettent de mettre en évidence une certaine continuité dans les représentations des

4 - Consultables sur les sites www.sisfrance.net, www.bdmvt.net et www.bdcavite.net. 
phénomènes. Ainsi les représentations actuelles des phénomènes établissent-elles encore parfois un lien, déjà relevé au XVIIe siècle, entre phénomènes météorologiques et séismes (Peltier, 1998). Quant à la rupture de "poches d'eau ", jadis avancée pour expliquer les inondations, elle se retrouve aujourd'hui, parfois sous une forme détournée (rupture ou délestage de barrage), dans le discours des inondés.

\subsection{Vivre avec le risque : pratiques vernaculaires et techniques de mitigation}

Les archives renseignent également sur la façon dont les sociétés anciennes intègrent, gèrent le risque. Jusqu'au XIXe siècle, les travaux de protection sont souvent élaborés par les communautés locales (voir par exemple Cœur, 2002), sauf cas relativement rare d'intervention des pouvoirs centraux (monarchie de Savoie en particulier - Girel, 1993 ; Vassalo, 2002). Les sources privilégiées sont donc les archives communales (séries relatives aux cours d'eau, aux chemins ou, souvent, plus largement aux travaux) ou, souvent, les archives des groupements de particuliers ou de communes (syndicats en France, bourgeoisies en Suisse, consorteries en Vallée d'Aoste, par exemple). Plus fréquemment, la prise en compte du risque se fait par les pratiques constructives ou d'aménagement du territoire. Dans le premier cas, il s'agit par exemple de dispositions architecturales visant à réduire la vulnérabilité des bâtiments: granges foraines protégées des avalanches par des forts de pierre sèche dans la vallée de Barèges (Pyrénées centrales ; Barrué-Pastor, Barrué, 1998) ou en Suisse (Coaz, 1910 ; Schoeneich, Busset-Henchoz, 1998); étables souterraines ("crottes») n'offrant pas de prise aux avalanches en Vallée d'Aoste (Remacle, 1991). L'analyse de l'architecture vernaculaire constitue alors une source précieuse pour comprendre comment, dans les périodes anciennes, on faisait face aux risques. Dans le second cas, l'analyse de cartes anciennes ainsi que des travaux de terrain couplés à l'histoire des phénomènes permettent de comprendre comment les communautés ont arbitré, dans leurs choix d'implantation, entre les ressources, les contraintes et les risques de leur terroir. Dans le Comminges (Pyrénées centrales), par exemple, l'implantation des villages sur des cônes de déjection correspond non pas à une prise de risque qu'on pourrait juger excessive mais à un choix effectué selon les potentialités et les contraintes du terroir (fond de vallée inondable annuellement, cône inondable lors d'épisodes violents mais rares) et, à une échelle très fine, à une connaissance des sites permettant de limiter le risque grâce à un peuplement privilégié des zones les moins exposées du cône (Antoine, Desailly, 2001). On mesure bien, sur ce type de problématique, la nécessité de croiser des sources anciennes (données chronologiques, plans et cartes anciens), des données actuelles (études de terrain) et des investigations géoarchéologiques pour parvenir à une bonne compréhension des choix sociaux effectués en matière de risque.

Au cours de la période récente (XIXe-XXe siècles), les données les plus fournies sont celles des services de l'Etat, celui-ci ayant souvent pris le relais des collectivités locales dans la réalisation de travaux de protection et le contrôle du territoire (PSS, PPR, etc.). Là encore, l'étude des données anciennes permet de mieux comprendre les aménagements récents, qui sont souvent conçus en référence aux travaux anciens. L'un des plus beaux exemples est la Troisième correction du Rhône en Valais, qui consiste en un aménagement visant à corriger les défauts des «corrections» précédentes survenues à la fin du XIXe siècle et dans les années 1940-1960. L'analyse des archives cantonales du Valais, complétées par les archives communales de la ville de Sion, très fournies sur ce sujet (série relative aux travaux publics) montre que chacune des " corrections » a été mise en place en réaction aux défauts de la précédente ; la troisième en date (en cours de réalisation) visant à redonner de l'espace au cours d'eau pour améliorer à la fois sa capacité d'écoulement des eaux de crue et sa qualité biologique (Peltier, 2005). 


\subsection{Les politiques publiques de gestion du risque}

L'analyse des politiques publiques anciennes obéit aux mêmes règles que celle des dispositifs en vigueur : analyse des conditions d'élaboration de la politique publique (discours associés), de son effectivité (le dispositif est-il mis en œuvre et comment ?), de son efficience (quels sont les effets induits par ce dispositif ? les moyens associés sont-ils adaptés aux fins visées ?) et de son efficacité (le dispositif atteint-il son but?). Le colloque organisé en 2001 à Grenoble sur les pouvoirs publics face aux risques a contribué à faire le point sur cette question (Favier, 2002).

La difficulté tient au caractère parfois lacunaire des informations disponibles. La matière première (textes de loi, décrets, etc.) a été conservée sur la longue durée et est facilement accessible. En revanche, l'analyse de la mise en œuvre de la politique peut être plus compliquée. Les sources disponibles sont de plusieurs ordres. Tout d'abord, du côté de l'Etat, les rapports des acteurs chargés de mettre en place les politiques de gestion des risques : préfets, agents des Eaux et Forêts et des Ponts et Chaussées, qui relatent les réussites et les échecs des règlementations. Mais aussi, du côté des destinataires des politiques publiques, les élus locaux, les organisations collectives telles que les syndicats de vallée dans les montagnes pyrénéennes, et les habitants. Grâce d'une part aux délibérations communales, d'autre part aux pétitions et autres lettres de protestation conservées dans les archives préfectorales, on connaît bien les grandes difficultés rencontrées par la politique de reboisement des montagnes à partir des années 1860 (Corvol, 1987 ; Larrère, 1992 ; Subotsch, 1999).

\subsection{La construction de la vulnérabilité des sociétés}

Entendue au sens d'exposition à un phénomène, la vulnérabilité se construit dans le temps, au fur et à mesure que les sociétés aménagent, modifient un territoire. Les données cartographiques anciennes permettent de reconstituer l'accroissement progressif de cette exposition, d'en comprendre les principales phases, mais aussi, dans une perspective plus opérationnelle, de qualifier la vulnérabilité de certains quartiers en fonction de leur époque et donc de leurs caractéristiques de construction (Guéguen et al., 2008). Les cadastres napoléoniens puis les cartes topographiques constituent les données les plus anciennes, certains pays comme la Suisse disposant par exemple de cartes topographiques au 1/50 000 de belle qualité dès les années 1880 (fig. 4). Les données photographiques (prises de vue paysagères, par exemple) et, plus anciennement, iconographiques, peuvent permettre de compléter ces informations et de reconstituer la construction temporelle de la vulnérabilité.

Définie comme l'incapacité d'une société à faire face à un aléa, au niveau collectif ou au niveau individuel, la vulnérabilité actuelle peut également être mieux comprise grâce à l'étude des sources historiques. Certains freins à l'application des politiques publiques trouvent ainsi leur origine dans un passé plus ancien, pouvant contribuer à expliquer une certaine vulnérabilité institutionnelle. La difficile application des mesures de reboisement des montagnes, évoquée plus haut, trouve ainsi des résurgences dans l'application des PPR, dénoncés par les collectivités locales et les propriétaires concernés comme une ingérence de l'Etat dans les affaires de la commune, tout comme la politique RTM a été considérée au XIXe siècle comme une mainmise de l'Etat jacobin sur les territoires de montagne. Sans doute certaines difficultés actuelles peuvent-elles ainsi s'expliquer, en partie, par un lourd passé d'affrontement. Notons qu'en Suisse, une tradition davantage fondée sur le dialogue et le consensus dans le domaine de la gestion des risques émerge également des études historiques et se retrouve à l'heure actuelle (Peltier, 2005). 


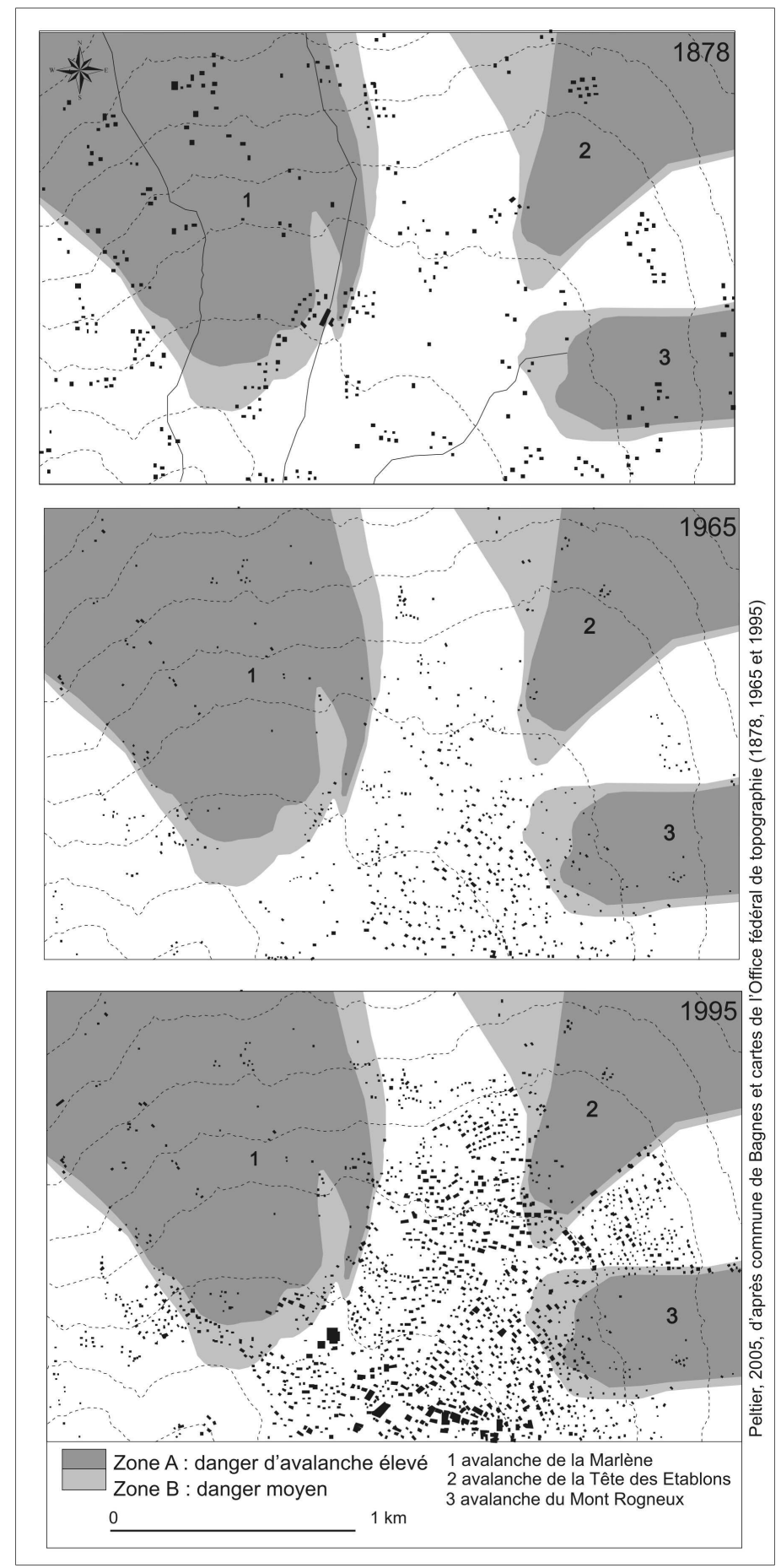

Figure 4. La construction progressive de la vulnérabilité : l'exemple de la station de ski de Verbier, Valais, Suisse, entre 1880 et 1995. Peltier, 2005

\section{Conclusion}

Récemment redécouvertes, les sources archivistiques ont été incontestablement le support d'un renouvellement des recherches sur les risques naturels en géographie, stimulées à la fois par l'émergence des problématiques liées à l'histoire de l'environnement, et par les besoins des gestionnaires du risque. Chronologiquement, l'approche géohistorique a fait en quelque sorte le lien entre les approches aléa-centrées classiques de la discipline jusque dans les années 1980 et les approches socio-centrées qui se sont développées beaucoup plus récemment. L'histoire des 
risques et des catastrophes s'intéresse en effet aussi bien aux processus biophysiques (nature des phénomènes, contextes écologiques, extension spatiale...) qu'aux postures sociales et sociétales qu'ils peuvent avoir suscitées.

Il n'en demeure pas moins que l'utilisation et l'analyse des sources historiques nécessitent des profils scientifiques hybrides, ou une collaboration des savoirs et savoir-faire scientifiques, car si les compétences traditionnelles des géographes ne suffisent pas à faire parler en toute objectivité et en toute efficacité les documents (maîtrise nécessaire des contextes politiques, institutionnels, sociologiques, économiques), les compétences de l'historien spécialiste de l'archive ne suffisent pas non plus (difficultés à interpréter par exemple le type de processus biophysique en jeu dans une catastrophe et la signification de son contexte spatial et territorial).

Au-delà de ces considérations générales, plusieurs écueils et limites à l'utilisation des sources historiques subsistent. On insistera d'une part sur la difficulté persistante à traduire quantitativement et à valider les informations relatives aux événements passés. D'autre part, richesse et précision des sources diminuant logiquement en remontant dans le temps, le défi d'une continuité de l'information entre les données archivistiques et les données paléoenvironnementales et géoarchéologiques est toujours d'actualité. Pourtant, à l'heure du développement durable et des interrogations liées au changement climatique, à l'heure des scenarii environnementaux prospectifs auxquels ils donnent lieu, la validation des données historiques et la reconstitution la plus ancienne possible des dynamiques environnementales passées sont fondamentales.

\section{Bibliographie}

ANTOINE J.-M., 1992, La catastrophe oubliée. Les avatars de l'inondation, du risque et de l'aménagement dans la vallée de l'Ariège (Pyrénées françaises, fin XVII $-X X^{e}$ siècles), Thèse de Doctorat Géographie, Université de Toulouse-Le Mirail, 495 p.

ANTOINE J.-M., DESAILLY B., 2000, Etude de faisabilité de bases de données historiques sur les inondations Base nationale et bases départementales, rapport, 41 p., base de données informatisée Accès ${ }^{\mathrm{TM}}$ (Microsoft) sur le département du Gers. Toulouse, GEODE UMR-5602 CNRS, Lyon, CERTU et Ministère de l'Environnement et de l'Aménagement du Territoire.

ANTOINE J.M., DESAILLY B., 2001, Habitat, terroirs et cônes de déjection torrentiels dans les Pyrénées commingeoises, in M. Berthe et B. Cursente ed., Villages pyrénéens - Morphogenèse d'un habitat de montagne, Université de Toulouse-Le Mirail, p. 27-44.

ANTOINE J.-M., DESAILLY B., GAZELLE F., 2001a, Les crues meurtrières, du Roussillon aux Cévennes, Annales de Géographie, vol. 110, n622, p. 597 623.

ANTOINE J.-M., DESAILLY B., HURAND A., LANUSSE M., 2001b, Méthodologie de recherche historique pour une cartographie des risques naturels dans les Pyrénées Application à une zone test comprenant les communes de la zone montagne du département de la Haute-Garonne, rapport et base de données informatisée Accès ${ }^{\mathrm{TM}}$ (Microsoft). Toulouse, DIREN Midi-Pyrénées, Délégation RTM
«Massif Pyrénées», GEODE UMR-5602 CNRS, Université de Pau et des Pays de l'Adour.

ANTOINE J.-M., 2009, L'histoire du climat par ses extrêmes. Sources géohistoriques et inondations dans les Pyrénées depuis le Petit Age Glaciaire, Actes de la table-ronde "Le Petit Age Glaciaire dans le Sud de la France: impacts morphogénique et sociétaux 》, Musée Archéologique, Lattes, 11 mai 2007, à paraittre in Archéologie du Midi Médiéval.

ARNAUD-FASSETTA G., 1998, Dynamiques fluviales bolocènes dans le delta du Rhône, thèse de doctorat, Université d'Aix-Marseille 1, Lille, Presses Universitaires de Septentrion, 329 p.

BARAILLE S., 2001, Les crues dommageables dans le bassin de la baute Durance (Hautes-Alpes, France). Recensement depuis le XIVe siècle; signification climatique; facteurs météorologiques et prévision, thèse de doctorat, Université de Savoie, $400 \mathrm{p}$.

BARRUE-PASTOR M., BARRUE M., 1998, Mémoire des catastrophes, gestion des risques et architecture paysanne en montagne. L'exemple des vallées du HautLavedan dans les Pyrénées centrales françaises, Revue de Géographie Alpine, n 2, p. 25-36.

BEROT M., 1998, La vie des hommes de la montagne dans les Pyrénées racontée par la toponymie, Milan, 389 p. 
BESSAT H., GERMI Cl., 1993, Lieux en mémoire de l'Alpe. Toponymie des alpages en Savoie et Vallée d'Aoste, Grenoble : ellug, 229 p.

BRIFFAUD S., 1993, Le savant, l'Etat et la catastrophe. L'émergence d'une nouvelle approche des sinistres à travers une relation du «prodigieux débordement » de 1678, in Risques et aménagement dans les Pyrénées. Actes du colloque international tenu à Foix et à Pau, 30-31 mai 1990 et 8-9 juin 1990, Les Cabiers de l'Isard, $\mathrm{n}^{\circ} 4$, p. $15-30$.

CHALVET M., 2000, L'invention de la forêt méditerranéenne de la fin du XVIIIe siècle aux années 1960, Thèse de doctorat d'histoire, Université Aix Marseille, 3 volumes, $707 \mathrm{p}$.

CHAMPION M., 1862-1868, Les inondations en France depuis le VTe siècle jusqu'à nos jours, Paris, Dunod, 6 vol., réédition Cemagref, 2000

COAZ J., 1910, Statistik und Verbau der Lawinen in den Schweizeralpen, Berne : éd. Stämpfli \& Cie (Département fédéral de l'Intérieur), $126 \mathrm{p}$.

CCEUR D. 2002, Des associations de propriétaires pour lutter contre l'inondation: les syndicats de riverains dans la plaine de Grenoble (vers 1750-vers 1930), in FAVIER, Les pouvoirs publics face aux risques naturels dans l'bistoire, p. 131-152.

CCEUR D. 2003, La maîtrise des inondations dans la plaine de Grenoble (XVII- XXème siècle): enjeux techniques, politiques et urbains, Thèse de Doctorat en Urbanisme et Aménagement, Grenoble, Université P. MendèsFrance.

COMBE C., 2008, Le SIG narratif, outil de territorialisation $\mathrm{du}$ risque: mise en perspective géohistorique du risque fluvial en milieu urbain et périurbain, in $\mathrm{La}$ mise en carte des risques naturels, Géorisques $\mathrm{n}^{\circ} 2$, Presses Universitaires de la Méditerranée, p. 23-30.

CORVOL A., 1987, L'homme aux bois. Histoire des relations de l'bomme et de la forêt, $X V I I^{e}-X X^{e}$ siècle, Fayard, 585 p.

CORVOL A., 1999, Les sources de l'bistoire de l'environnement, le XIXe siècle, co-éd. L'Harmattan Direction des Archives de France, 502 p.

CORVOL A., 2003, Les sources de l'bistoire de l'environnement, le XXe siècle, L'Harmattan, 756 p.

CUBIZOLE H., 1994, La Dore et sa vallée. Approche géohistorique des relations Homme/milieu fluvial. Publications de l'Université de Saint-Etienne, thèse de doctorat, Université Blaise Pascal, Clermont-Ferrand, 389 p.

CULMANN K., ESCHER DE LA LINTH, LANDOLT E., 1869, Rapports des commissions d'experts sur les causes et l'importance des dommages causés en 1868 par les inondations dans les Cantons d'Uri, de St. Gall, des Grisons, du Tessin et $d u$ Valais, Berne: Imprimerie de Ch.-J. Wyss, 209 p.
DESAILLY B., 1990, Crues et inondations en Roussillon. Le risque et l'aménagement. Fin du XVIIe siècle - milieu du XXe siècle, thèse de doctorat de géographie, Université de Paris X - Nanterre, 352 p.

DION R., 1961, Histoire des levées de la Loire, R. Dion éd., 312 p.

FANTHOU Th., KAISER B., 1990, Evaluation des risques naturels dans les Hautes-Alpes et la Savoie. Le recours aux documents d'archives et aux enquêtes, Bulletin de l'Association des Géographes Français, 4, p. 323339.

FAVIER R. (dir.), 2002, Les pouvoirs publics face aux risques naturels dans l'bistoire / Actes du colloque international sur l'bistoire des risques naturels, Grenoble, 22-24 mars 2001, Grenoble : Publications de la MSH-Alpes, 444 p.

FAVIER R., GRANET-ABISSET A.-M. (dir.), 2005, Récits et représentations des catastrophes depuis l'Antiquité / Actes du colloque "Le traitement médiatique des catastrophes: entre oubli et mémoire ", Grenoble, 10-11-12 avril 2003, Grenoble : Publications de la MSH-Alpes, 408 p.

GARRY G., 1994, Evolution et rôle de la cartographie dans la gestion des zones inondables en France, Mappemonde, Vol. X, 4, p. 10-16.

GARRY G., 1996, La protection des personnes et des biens: vers une prise en compte du risque d'inondation, in NEBOIT-GUILHOT R. et DAVY L. (dir.), Les Français dans leur environnement, Nathan, p. 348366.

GAUTIER E., 1992, Recherches sur la morphologie et la dynamique fluviale dans le bassin du Buëch (Alpes du Sud), Thèse de Doctorat de Géographie, Université de Paris $\mathrm{X}$ - Nanterre, 439 p.

GIREL J., 1993, Endiguements et colmatages sardes dans la vallée de l'Isère à l'aval d'Albertville, in Piquet (éd.), Le fleuve et ses métamorphoses, Actes du colloque international, Université Lyon III-Jean Moulin, Paris : Didier Erudition, p. 217-232.

GUEGUEN Ph., LUTOFF C., DAVOINE P.-A., TALIERCIO G., COTTON F., CARTIER S., 2008, Analyse de la vulnérabilité sismique dans un pays à sismicité modérée. Le cas de Grenoble, communication au colloque $V$ ulnérabilités sociétales, risques et environnement, Toulouse, 14-16 mai 2008.

JACOB N., 1997, La crue d'octobre 1940 dans la basse vallée du Tech (Roussillon), d'après les dossiers des sinistrés, Annales de Géographie, vol. 106, n596, p. 414424.

L'aiguat del 40. Inundacions catastrofiques $i$ politiques de prevencio a la Mediterrania nord-occidental, 1993, Actes du colloque de Vernet-les-Bains, 18-20 octobre 1990, Barcelona: Generalitat de Catalunya - Servei Geologic de Catalunya, $484 \mathrm{p}$. 
LAHOUSSE Ph., 1994, Recherches géomorphologiques et cartographie des aléas naturels dans la vallée de la Guisane, thèse de doctorat, Université de Lille, 422 p.

LAHOUSSE Ph., 1997, L'apport de l'enquête historique dans l'évaluation des risques morphodynamiques: l'exemple de la vallée de la Guisane (Hautes-Alpes, Briançonnais), Revue de Géographie Alpine, 1, p. 53-60.

LANG M., CCEUR D., LALLEMENT C., NAULET R., 1998, Valorisation de l'information historique pour la prédétermination du risque d'inondation : application sur le bassin du Guiers, Ingénieries $E A T, \mathrm{n}^{\circ} 16$, décembre, p. 3-13.

LARRERE R., BRUN A., et al., 1979, Les reboisements en montagne depuis l'Empire, INRA, Laboratoire d'Economie et de Sociologie rurales d'Orléans; Présenté au Symposium d'Histoire forestière IUFRO, Nancy, 25-28 septembre 1979, 95p.

LARRERE R., 1992, L'érosion, l'avalanche et le forestier, Le risque en montagne. Les réalités et les images, Actes du $116^{e}$ Congrès des Sociétés savantes, Editions du CTHS, p. 21-40.

LEONE F., VINET F. dir., 2008, La mise en carte des risques naturels - Diversité des approches, Actes du colloque GESTER «La cartographie des risques naturels », Montpellier, Presses Universitaires de la Méditerranée, $89 \mathrm{p}$.

LE ROY LADURIE E., 1967, Histoire du climat depuis l'An Mil, Paris, Flammarion.

Les catastrophes naturelles dans l'Europe médiévale et moderne, 1996, Actes des XVe Journées Internationales d'Histoire de l'Abbaye de Flaran, 10-12 septembre 1993, Etudes réunies par B. Bennassar, Toulouse: P.U.M, 272 p.

MESCHINET DE RICHEMOND N., 1997, Les inondations catastrophiques sur la bordure montagneuse $d u$ Roussillon: dégâts et sinistrés, Thèse de Doctorat de Géographie, Université de Paris X - Nanterre, 431 p.

METAILIE J.-P., 1987, The degradation of the Pyrenees in the Nineteenth century - an erosion crisis?, Proceedings of the First International Conference on Geomorphology, Manchester, 09/1985, V. Gardiner ed., Chichester, J. Wiley, 1987, Part. II, p. 533-544.

METAILIE J.-P. dir., 1991, Le torrent et le fleuve : risques, catastrophes et aménagement dans les Pyrénées et leur piémont. Fin $X V I I^{e}-X X^{e}$ siècles, Rapport ATP-PIREN CNRS, Toulouse, Université de Toulouse le Mirail-CIMA URA-366 CNRS, 321 p.

MIRAMONT C., GUILBERT X., 1997, Variations historiques de la fréquence des crues et de la morphogenèse fluviale en Moyenne Durance. Géomorphologie: relief, processus et environnement, $\mathrm{n}^{\circ} 4, \mathrm{p}$. 325-338.
MIRAMONT C., 1998, Morphogenèse, activité érosive et détritisme alluvial bolocènes dans le bassin de la moyenne Durance, thèse de doctorat, Université d'Aix-Marseille I, $286 \mathrm{p}$.

NAULET, R., 2002, Utilisation de l'information des crues historiques pour une meilleure prédétermination du risque d'inondation. Application au bassin de l'Ardèche à Vallon Pont-d'Arc et St-Martin d'Ardèche, thèse de doctorat, Université Grenoble I et INRS-ETE (Québec), 322 p.

PARDE M., 1953, Sur les inondations en Aquitaine spécialement dans le bassin de la Garonne : à propos de la grande crue de février 1952, Revue Géographique des Pyrénées et du Sud-Ouest, vol. 24, p. 163-257.

PAYRASTRE O., 2005, Faisabilité et utilité du recueil de données historiques pour l'étude des crues extrêmes de petits cours d'eau - Etude du cas de quatre bassins versants affluents de l'Aude, thèse de l'Ecole Nationale des Ponts-etChaussées, $390 \mathrm{p}$.

PEIRY J.-L., 1988, Approche géographique de la dynamique spatio-temporelle des sédiments sur un cours d'eau intramontagnard; l'exemple de la plaine alluviale de l'Arve (Haute-Savoie), thèse de Géographie et Aménagement, Université Jean Moulin, Lyon 3, 376 p.

PEIRY J.-L., 1989, La mappe sarde. Utilisation pour une étude historique de l'Arve, Revue de Géographie de Lyon, nº 4, p. 197-203.

PELTIER A., 1998, Le risque sismique dans les HautesPyrénées. Risque négligeable? Risque négligé? Mémoire de maîtrise, Université de Toulouse - le Mirail, 258 p.

PELTIER A., 2005, La gestion des risques naturels dans les montagnes d'Europe occidentale. Etude comparative du Valais (Suisse), de la Vallée d'Aoste (Italie) et des Hautes-Pyrénées (France), Thèse de Doctorat de Géographie, Université de Toulouse - Le Mirail, 741 p.

PFISTER Ch., 1988, Klimageschichte der Schweiz. $1525-$ 1860, Das Klima der Schweiz von 1525-1860 und seine Bedeutung in der Geschichte von Bevölkerung und Landwirtschaft, Bern et Stuttgart.

PICHARD G., 1995, Les crues sur le bas-Rhône de 1500 à nos jours. Pour une histoire hydro-climatique, Méditerranée, n 3-4, p. 105-116.

REMACLE C., 1991, La maison rurale en vallée d'Aoste (Bionaz, Oyace, Torgnon). Etude de géographie historique et sociale, Thèse de doctorat de géographie-aménagement : Université Joseph-Fourier, Grenoble I, 359 p.

Revue de Géographie Alpine, 1998, Représentations des risques naturels en montagne, $\mathrm{n}^{\circ} 2$, tome 86 .

REYT Ph., 2000, La représentation du risque dans l'imaginaire des altitudes, Revue de Géographie Alpine, $\mathrm{t}$. $88, n^{\circ} 4$, p. $35-46$.

Sources - Travaux historiques, 1993, Histoire des catastrophes naturelles, $\mathrm{n}^{\circ} 33$. 
SALVADOR P.G., 1991, Le thème de la métamorphose fluviale dans les plaines alluviales du Rhône et de l'Isère, Thèse de Doctorat de Géographie, Université de Lyon III, $496 \mathrm{p}$.

SAMIVEL, 1984, Hommes, cimes et dieux. Les grandes mythologiques de l'altitude et la légende dorée des montagnes à travers le monde, Paris : Arthaud, 378 p.

SCHOENEICH P., BUSSET-HENCHOZ M.-C., 1998, Les Ormonans et les Leysenouds face aux risques naturels. Représentation des risques naturels et stratégies d'occupation du territoire dans la Vallée des Ormonts (Préalpes vaudoises), Rapport final PNR 31, Zürich : vdf, 230 p.

SCHÜLE R.-C., 1992, La peur à la montagne, Le risque en montagne. Les réalités et les images, Actes du $11 \sigma^{e}$ Congrès des Sociétés savantes, Editions du CTHS, p. 65-74.
SOUTADE G., 1993, Les inondations d'octobre 1940 dans les Pyrénées-Orientales, Perpignan, Direction des Archives Départementales des Pyrénées-Orientales, 351 p.

SUBOTSCH N., 1999, Comparaison des politiques de gestion des forêts à rôle de protection dans les pays alpins, signataires de la Convention alpine, Thèse de doctorat: Laboratoire de la montagne alpine, Université Joseph Fourier, Grenoble, 484 p. + annexes.

SURREL A., 1841, 2édition 1870, Etude sur les torrents des Hautes-Alpes, Paris: Dunod, 317p., réédité par Nîmes : Lacour, 2002.

VASSALO N., 2002, La monarchie de la maison de Savoie face aux catastrophes naturelles: les «atti di visita » pour les ravinements et tempêtes, in FAVIER, Les pouvoirs publics face aux risques naturels dans l'bistoire, p. 105-129.

VOGT J. (dir.), 1979, Les tremblements de terre en France, Editions du BRGM, Mémoire nº9, 225 p. 